\title{
High Temperature Surface Conductivity of Hydrogenated Diamond Films Exposed to Humid Air
}

\author{
K. SteC ${ }^{a}$, P. SzRoeder ${ }^{a, *}$ And K. Benzhour ${ }^{b}$ \\ ${ }^{a}$ Institute of Physics, Nicolaus Copernicus University, Grudziądzka 5/7, 87-100 Torun, Poland \\ ${ }^{b}$ Department of Bases of Theoretical Biomedical Studies and Medical Computer Science, Collegium Medicum, \\ Nicolaus Copernicus University, Jagiellońska 13, 85-067 Bydgoszcz, Poland \\ Surface conductivity of thin diamond films was measured as a function of temperature up to $450{ }^{\circ} \mathrm{C}$. \\ Hydrogenated diamond was synthesized by chemical vapor deposition in hydrogen/carbon plasma. Low values \\ of charge carrier activation energy $(\approx 10 \mathrm{meV})$ were observed, when hydrogenated diamond films were exposed \\ to the ambient humid air. However, the activation energy increased by two orders of magnitude as film \\ temperature exceeded $300^{\circ} \mathrm{C}$. We have attributed this behavior to the desorption of the $\mathrm{H}_{2} \mathrm{O}$ adlayer. The jump \\ of the activation energy did not occur, when experiment was performed in vacuum. We have also shown that \\ donor doping leads to the up-shift of the Fermi level much above the acceptor-like band gap levels induced by \\ surface C-H bonds, which cannot be compensated by transfer of electrons from diamond to the double $\mathrm{H}-\mathrm{H}_{2} \mathrm{O}$ layer.
}

PACS numbers: 73.25.+i, 81.05.ug

\section{Introduction}

Devices based on diamond films grown by the chemical vapor deposition (CVD) process are drawing much attention due to the electronic properties of diamond material, such as the high thermal conductivity, large band-gap, high charge carrier mobility [1]. This makes the CVD diamond good candidate for construction of high power and high frequency semiconductor devices [2].

Several studies have shown that CVD diamond could enable the fabrication of materials exhibiting electrical properties similar to those of some of the best natural gems. Numerous experiments have shown that hydrogen-terminated CVD diamond reveals a $p$-type conductivity. Two-dimensional hole conductivity is an interesting phenomenon for surface science [3], as it can be applied for fabrication of novel electronic devices such as field-effect transistors, chemical sensors and biosensors [4-6].

Among several models proposed in the literature to explain the surface $p$-type conductivity, the electrochemical transfer doping model $[7,8]$ has attracted the most attention. It is based on the observation that negative electron affinity of the hydrogen terminated diamond surface induces a low ionization energy which favors the electron transfer from the valence band to physisorbed adsorbates. The surface conductivity is then promoted by exposing the hydrogen-terminated diamond surface to humid air. The layer of water, which spontaneously forms on the surface, induces a subsurface hole accumulation layer with the hole concentration of up to $10^{13} \mathrm{~cm}^{-2}$ [9].

\footnotetext{
* corresponding author; e-mail: psz@fizyka.umk.pl
}

\section{Experimental setup}

The hydrogenated diamond (HD) films were fabricated by the hot filament CVD method on the (100) silicon substrate. The substrate temperature controlled by the thermocouple wire was set to $750{ }^{\circ} \mathrm{C}$. The electrically heated tungsten filament positioned $6 \mathrm{~mm}$ under the substrate surface was used to activate the $\mathrm{H}-\mathrm{C}$ plasma. The pressure and flow rate of the feed gas mixture (propane, butane and $\mathrm{H}_{2}$ ) were kept constant during all the deposition experiments. The deposition time was $24 \mathrm{~h}$. For fabrication of N-doped diamond, nitrogen was added to the gas mixture. The growth parameters are collected in Table. As the synthesis process took place in the hydrogen/carbon plasma, we can assume that both the undoped and the N-doped diamond layers are hydrogen terminated.

TABLE

Deposition process parameters.

\begin{tabular}{c|c|c|c|c}
\hline \hline Sample & $\begin{array}{c}\text { Propane-butane } \\
\text { [sccm] }\end{array}$ & $\begin{array}{c}\text { Hydrogen } \\
\text { [sccm] }\end{array}$ & $\begin{array}{c}\text { Pressure } \\
\text { [Torr] }\end{array}$ & $\begin{array}{c}\text { Nitrogen } \\
\text { [sccm] }\end{array}$ \\
\hline S1 & 8 & 400 & 30 & 0 \\
S2 & 8 & 400 & 30 & 30
\end{tabular}

The characteristics of the diamond films were examined using a scanning electron microscopy (SEM), X-ray diffraction (XRD) and electrical surface conductivity. The electrical characteristics were measured in a flat configuration using Keithley 6517 electrometer with a 2 -point contact probe made from very thin platinum wire kept at a distance of approximately $1.9 \mathrm{~mm}$. The measurement chamber was evacuated after samples were inserted in the specimen stage. Then 2-3 heating cycles 
started, up to $450{ }^{\circ} \mathrm{C}$, which were accompanied with collection of the resistance data. To observe the influence of water adsorption on temperature behavior of resistance in each sample, the last measurement cycle was performed after we had got air in the measurement chamber.

\section{Results and discussion}

The morphology of the CVD diamond layer is shown in Fig. 1. SEM images reveal the continuous structure of diamond layers. In case of the undoped sample S1, well-faceted crystalline grains with a predominance of (100) crystalline surfaces are seen. On the other hand, predominance of well-faceted crystalline grains with angular shapes corresponding to (111) crystalline surfaces is characteristic of the N-doped diamonds (sample S2). The size of the crystallite grains decreases, when $\mathrm{N}$ atoms are introduced into the structure.
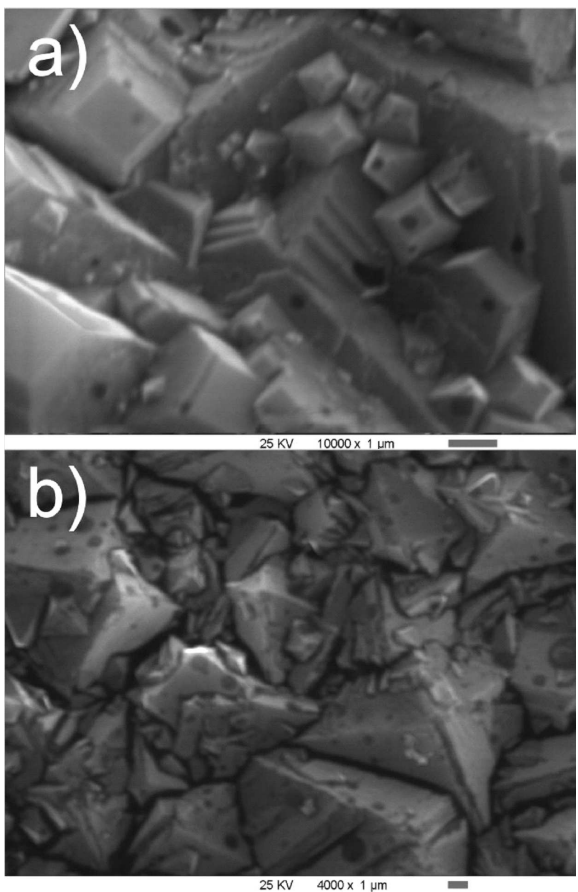

Fig. 1. SEM images of deposited diamond films: (a) sample S1, (b) sample S2.

The Raman spectra of diamond layers are displayed in Fig. 2. For both samples a peak at $1332 \mathrm{~cm}^{-1}$ is observed. This line is attributed to the first order diamond $s p^{3} \mathrm{Ra}$ man peak. The diamond peak position is blue-shifted according to perfect diamond proving compressive stress in crystallite grains. The broad band at about $\approx 1540 \mathrm{~cm}^{-1}$ corresponds to the graphitic $s p^{2}$ phase. Let us note that doping with nitrogen leads to an increase in the intensity of graphitic band. As it is seen on the Raman spectrum of the N-doped diamond, the weak luminescence background is present proving higher concentration of defect states.

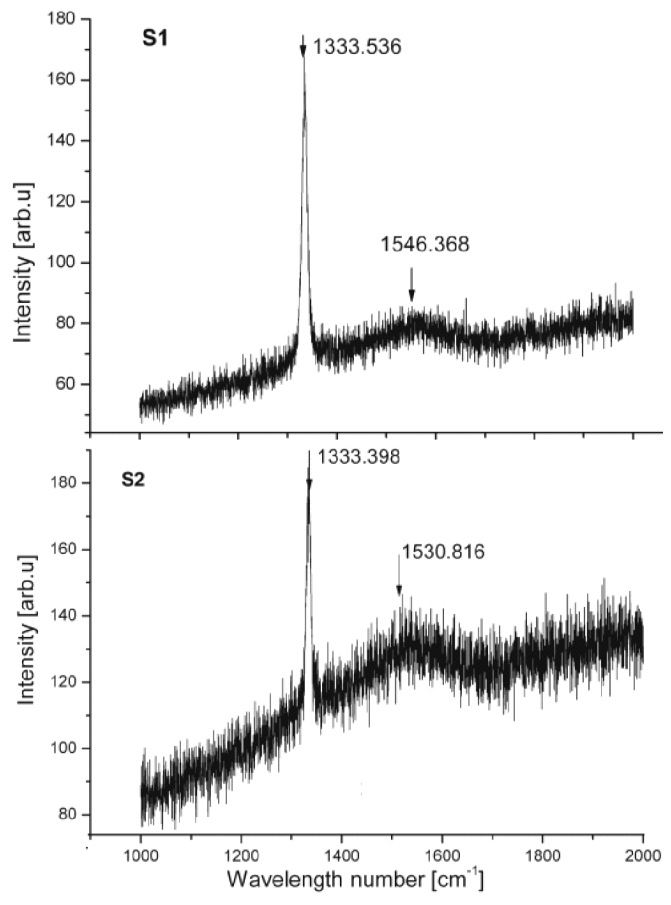

Fig. 2. Raman spectra of samples S1 and S2.

The temperature dependences of surface resistance measured in vacuum and the ambient air are shown in Fig. 3. The resistance of both the undoped and the $\mathrm{N}$-doped diamond films falls down with increasing temperature. The semiconductor behavior allows us to estimate the activation energy of current carriers from the $R(T)$ dependence

$$
R \cong R_{0} \exp \left(\frac{E_{\mathrm{a}}}{k T}\right),
$$

where $R$ is resistance, $R_{0}$ is the parameter depending on the material, $E_{\mathrm{a}}$ is the activation energy, $k$ is the Boltzmann constant, and $T$ is the absolute temperature. The

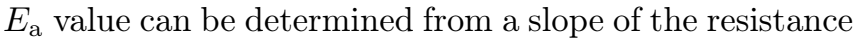
curve drawn in the Arrhenius plot.

As it is seen in Fig. 3a, electrical surface resistance of the undoped diamond is very sensitive to normal humid air. During the first heating cycle performed in vacuum we observed the resistance changes with very low activation energy of $10 \mathrm{meV}$. The characteristic feature of the temperature resistance dependence is the change of the Arrhenius plot slope at $300{ }^{\circ} \mathrm{C}$ that corresponds to jumping of the activation energy from $10 \mathrm{meV}$ to $\approx 1.5 \mathrm{eV}$.

On the other hand, the temperature dependence of the surface resistance in vacuum did not change during repeated heating cycles. The $E_{\mathrm{a}}$ value was almost constant in the measured temperature range and varied between $200 \mathrm{meV}$ and $300 \mathrm{meV}$. However, the temperature resistance character changed when we got again air in the chamber. Similarly to the first heating cycle, the activation energy of the charge carriers became very low (about $50 \mathrm{meV}$ ) in the range of temperature between room tem- 

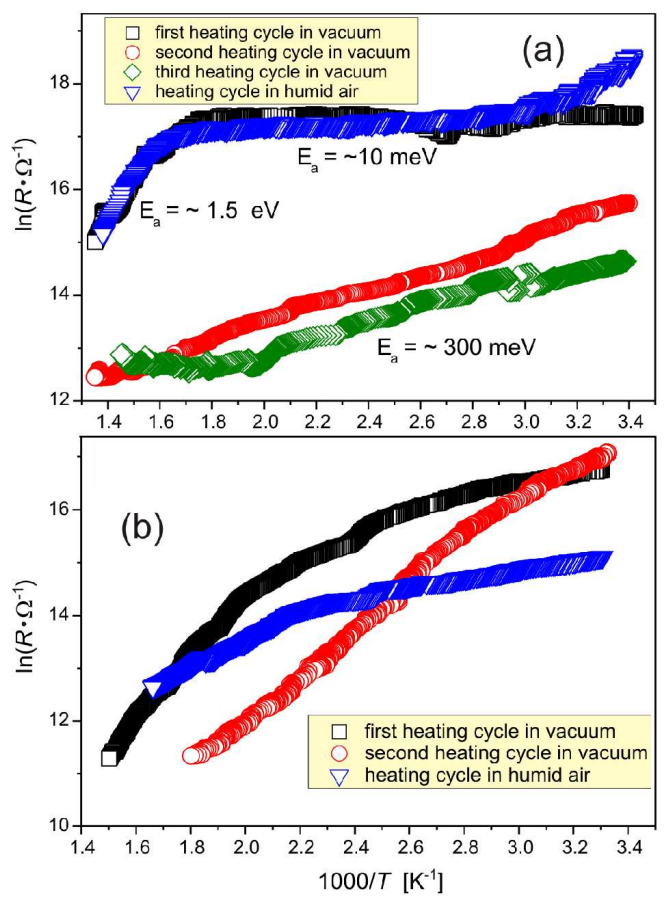

Fig. 3. Temperature dependence of surface resistance of the undoped (a) and N-doped (b) diamond films.

perature and $300{ }^{\circ} \mathrm{C}$ and increased rapidly above $300{ }^{\circ} \mathrm{C}$.

These results imply that surface electrical resistivity is under strong influence of such factors as sorption process of molecules of water from humid air and charge transfer from diamond to the $\mathrm{H}$-atoms induced by water adlayer (see Fig. 4a). During the first cycle of heating in vacuum, the sample still contained the adsorbed water. The change of the Arrhenius plot slope at $300{ }^{\circ} \mathrm{C}$ corresponds to the water desorption process accompanied with rapid increase in the activation energy. Let us note that this temperature is equivalent to the energy of $\approx 0.5 \mathrm{eV}$. The calculated value of binding energy of water adlayer is $0.12 \mathrm{eV}$ and $0.88 \mathrm{eV}$ for $\mathrm{H}_{2} \mathrm{O}$ and $\mathrm{H}_{3} \mathrm{O}^{+}$, respectively [10].

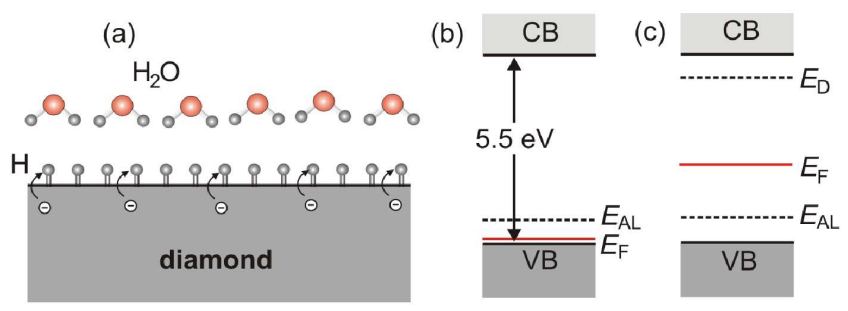

Fig. 4. (a) Electrostatic surface dipole layer formed due to the adsorption of $\mathrm{H}_{2} \mathrm{O}$. (b) Electron energy diagram of hydrogenated diamond. The $\mathrm{CB}$ and $\mathrm{VB}$ are conduction and valence band, respectively. Acceptor-like gap states induced by dangling bonds terminated with hydrogen above the valence band are marked as $E_{\mathrm{AL}}, E_{\mathrm{F}}$ is the Fermi level. (c) Effect of the N-doping on the $E_{\mathrm{F}}$ shift according to the electronic bands. The donor states are marked as $E_{\mathrm{D}}$.
The Arrhenius plots of the N-doped diamond differ essentially from that of the undoped samples. First of all, during first heating cycle in vacuum we had observed relatively high activation energy, about $150 \mathrm{meV}$. Its value started slowly to increase at temperature of $150^{\circ} \mathrm{C}$ and reached value of $\approx 450 \mathrm{meV}$ at $300^{\circ} \mathrm{C}$. We had observed similar behavior during the second cycle of heating in vacuum with activation energies of $\approx 200 \mathrm{meV}$ and $\approx 900 \mathrm{meV}$, respectively. When we got air in the chamber, we obtained almost constant activation energy (about $700 \mathrm{meV}$ ) in the whole measured range of temperatures.

The results obtained for donor doped diamond demonstrate a doping to be another important factor for the surface electrical transport properties. As donor doped diamond has excess of negative carriers which cannot be compensated by electron transfer from diamond to $\mathrm{H}-\mathrm{H}_{2} \mathrm{O}$ double layer, we did not observe the influence of water adsorption on the resistivity characteristics.

To explain the changes of activation energy of charge carriers with temperatures, in Fig. $4 \mathrm{~b}$ and $\mathrm{c}$ we have shown the electronic diagrams of the undoped and $\mathrm{N}$-doped diamond films. In both cases there are formed acceptor-like gap states, $E_{\mathrm{AL}}$, above the valence band. However in the former case the transfer of electrons from the diamond film to the H-atoms leads to the downshift of the Fermi level below the $E_{\mathrm{AL}}$. In such case we need comparatively low energy to excite electrons from valence band to the localized acceptor-like states and to induce the electrical hole conductivity. The desorption process results in upshift of the Fermi level above the $E_{\mathrm{AL}}$ and increase in the activation energy. In the latter case the N-doping causes the strong upshift of the Fermi level which could not be compensated by the charge transfer from diamond to the $\mathrm{H}-\mathrm{H}_{2} \mathrm{O}$ double layer.

\section{Conclusions}

The increased surface conductivity of hydrogenated diamond films is a well known but not fully understood phenomenon. As we have shown, temperature measurements performed in vacuum and in ambient air allow us to observe the influence of adsorption and desorption process on the surface resistance characteristics. In particular, we have estimated the activation energy of the charge carriers. Its value in undoped samples increases by two orders of magnitude at $300{ }^{\circ} \mathrm{C}$ indicating the destruction of the $\mathrm{H}-\mathrm{H}_{2} \mathrm{O}$ double layer and transfer of electrons to the diamond film. However this process is reversible. Such significant changes of the resistance character are not observed in donor doped diamond.

\section{References}

[1] K. Larsson, J. Ristein, J. Phys. Chem. B 109, 10304 (2005).

[2] C.E. Nebel, H. Kato, B. Rezek, D. Shin, D. Takeuchi, H. Watanabe, T. Yamamoto, Diamond Relat. Mater. 15, $264(2006)$. 
[3] J. Ristein, M. Riedel, F. Maier, B.F. Mantel, M. Stammler, L. Ley, J. Phys., Condens. Matter 13, 8979 (2001).

[4] F. Maier, M. Riedel, B.F. Mantel, J. Ristein, L. Ley, Phys. Rev. Lett. 85, 3472 (2000).

[5] J. Ristein, Surf. Sci. 600, 3677 (2006).

[6] L. Ley, J. Ristein, F. Maier, M. Riedel, P. Strobel, Physica B 262, 376 (2006).

[7] J. Ristein, J. Phys. D, Appl. Phys. 39, R71 (2006).
[8] J. Ristein, P. Strobel, L. Ley, Adv. Sci. Technol. 48, 93 (2006).

[9] V. Chakrapani, J.C. Angus, A.B. Anderson, S.D. Wolter, B.S. Stoner, G.U. Sumanasekara, Science 318, 1424 (2007).

[10] A.N. Andriotis, G. Mpourmpakis, E. Richter, M. Menon, Phys. Rev. Lett. 100, 106801 (2008). 